\title{
Toward Theoretical and Experimental Synergies in Neuroscience: A Personal View
}

\author{
Frances K. Skinner
}

Keywords: Synergy, neuroscience, theory, experiment

doi:10.1017/cjn.2017.215

Can J Neurol Sci. 2017; 44: 747-748

When I was invited to chair a plenary symposium at the Canadian Association for Neuroscience (CAN) meeting in 2016 with Larry Abbott as the featured plenary speaker, I happily accepted. This was not only because it was a privilege, but also because it was an opportunity to highlight my views from the perspective of my chosen symposium title, "Toward Theoretical and Experimental Synergies in Neuroscience." Larry and Eve Marder have shaped my views from my time at Brandeis University and over the years.

\section{Collaborations NeEded}

It goes without saying that neuroscience research today requires collaborations. More specifically, fruitful integration of theory and experiment is strongly needed to move us toward an understanding of brain function, but the way forward is not clear-cut and needs to be forged. ${ }^{1}$ Despite this need, theoretical and experimental studies in neuroscience often end up being separate endeavours. In large part, this is due to the complexity of biological systems and their wide-ranging temporal and spatial scales, so that particular choices need to be made when using theoretical or experimental approaches. Another reason could be because theoretical and experimental approaches focus on different "Aristotelian" causes (material, efficient, formal, final) to explain phenomena, ${ }^{2}$ and unless integrated, theory and experiment remain independent of each other. To help overcome this separation, in an eLife journal editorial we suggested that theoretical papers could be written so as to be more accessible, that more complete experimental datasets and explanations could be provided, and that more theoretical and mathematical techniques could be given in college and university biological programs. $^{3}$

\section{More Conversations NeEded}

In a recent report, ${ }^{4}$ I came across the following statement: "For collaboration to happen, research not only needs to be discoverable, it also needs to be understood" (p. 22). To me, this statement captures the main obstacle to creating synergies between theory and experiment: there are not enough ongoing conversations between theorists and experimentalists for understanding and thus synergies to emerge.

\section{Challenges and considerations}

Based on my observations and experiences to date, I think that present-day challenges to theoretical and experimental synergies mainly stem from fundamental differences in social working styles and efforts.

"Because science starts with human interactions: if we want theory and experimental neuroscience to strengthen each other, we must hope for people with different cultures, expertise, perspectives, and footwear to leave their prejudices at the door and learn to better appreciate each others' strengths . . . and where sociological forces must be tamed." Gilles Laurent's words resonated with me when I read them in 2000, and even more so today now that I have some experience and an appreciation for practical working differences among graduate students and faculty in physical sciences (e.g., math, engineering) or in life sciences (e.g., physiology, biology). For a start, the style and number of graduate courses differ, and mandatory teaching/ tutoring requirements differ. Another obvious difference between theoretical and experimental work is the contrasting types of efforts and techniques that are used. For example, for theoretical work: writing, testing and debugging computer codes, using dynamical systems analyses, installing and upgrading software, or simply theorizing; versus for experimental work: recording and stimulating individual cells in a biological circuit, using two-photon calcium imaging, doing dissections and surgeries, or simply designing experimental protocols.

Research is clearly not a 9-to-5 job. However, lab experiments typically occur during the day within the lab, whereas theoretical work can typically occur at any time or place (with the internet) and often is in full swing during the wee hours of the morning. This leads to widely varying working hours, which can make it difficult for regular interfacing and conversations to occur. As well, an appreciation of how much time and effort is involved in a particular research task may not be realized. Of course, how much time and effort is required is also a matter of technical proficiency

From the Krembil Research Institute, University Health Network, Toronto, Canada (FKS); the Departments of Medicine (Neurology) and Physiology, University of Toronto, Toronto, Canada (FKS).

Received April 10, 2017. Date of Acceptance May 8, 2017.

Correspondence to: Frances K. Skinner, Krembil Research Institute, 60 Leonard Avenue, Krembil Discovery Tower, 4KD510, Toronto, Ontario M5T 2S8, Canada.

E-mail: frances.skinner@utoronto.ca OR frances.skinner@uhnresearch.ca 
and experience. Overall, a strong dose of open-mindedness and critical questioning is required along with mutual respect to help ensure that conversations start and continue.

It is important to note that collaborations get initiated when curiosity is piqued, as can happen during random trainee conversations. As I found out during my time at Brandeis, the outstanding collaboration that developed between Larry and Eve was initiated by trainee interactions in their respective labs.

So, at the end of the day, it is about human interactions. We need to ensure that we have lots of times and spaces for regular discussions between theoreticians and experimentalists to occur, and that the above-mentioned differences do not prevent this. This could occur through institutional and interdepartmental coffee breaks and promotion of dynamic interdisciplinary meetings and workshops, for example.

In the same report ${ }^{4}$ it was noted that, "Collaborations can be hugely beneficial for science, but there is some evidence that they can have a detrimental effect on the careers of some individuals" (p. 7). Having regular interactions and conversations would help remove detrimental effects, as there would be a chance to develop an appreciation and understanding of the specifics of each other's work and perspective, thus benefiting the individual. Moving beyond articles and authorships are part of the ongoing process incentivizing collaborative discussions (pp. 8-9). Of course, if the theorist and experimentalist were one and the same person, then these challenges would be eliminated. However, the high level of expertise in today's sophisticated and wide-ranging experimental and theoretical approaches makes this unlikely. More possible is that the same person has experienced both theoretical and experimental approaches and can thus help address synergy challenges.

In essence, we need to recognize fundamental differences in theory and experiment and appreciate different disciplinary cultures so as to overcome human interaction challenges. In this way, more opportunities for conversation can occur in the first place.

\section{IT'S ALL ABOUT THE INDIVIDUAL}

For theoretical and experimental synergies to occur, it is ultimately about the passion and perseverance of the individual. We get excited about different things, are driven by and are passionate about different things, and it is these differences that make research life interesting. There just has to be some overlap for it to become a synergistic collaboration.

An example. My very first $\mathrm{PhD}$ student wanted to jump into the detailed multi-compartment modeling world, partly because her artistic side was drawn to the morphological beauty of individual neurons. I initially resisted from mathematical modeling considerations, but at the time it was also becoming clear that biophysical specifics in the dendrites of inhibitory cells of the hippocampus could not be ignored. This latter aspect combined with her determination led to collaborations being developed with experimentalists excited about these particular cells-clear overlapping interests. This initial collaboration has fuelled subsequent synergistic collaborations.

We need to nurture the individual passion and be aware of what underlies it - passion and dedication cannot be forced. Further, it is helpful to know what underlies an individual's passion, as this can lead to the development of synergies and the formulation of common drives and goals.

For myself, the drive has always been about combining math and biology, and I have experienced aspects of both theoretical and experimental approaches. My passion is to have the mathematical models and the experimental data work in harness so as to contribute to an understanding of neurological disease. As such, I feel fortunate to be working in a hospital research institute doing mathematical modeling - it is personally motivating!

\section{Closing Comments}

The essence of my commentary that expands on my presentation at the CAN2016 plenary symposium is that for theory and experiment to synergize there needs to be more regular discussions and interactions between individual theorists and individual experimentalists. This takes time and open-mindedness. We need to realize and recognize each other's hard work, dedication, and perspective. That is, we need to listen to each other, ask lots of critical questions, and always start with mutual respect.

My personal view is that it's not mainly about the money and the scenery, although they are of course needed! I believe that an emphasis on the passionate individual and an appreciation of disciplinary differences trumps all in moving toward theoretical and experimental synergies in neuroscience.

\section{DisClosures}

Frances Skinner does not have anything to disclose.

\section{REFERENCES}

1. Churchland AK, Abbott LF. Conceptual and technical advances define a key moment for theoretical neuroscience. Nat Neurosci. 2016;19(3):348-9.

2. Brette R. What is computational neuroscience? (XVI) What is an explanation? Romain Brette: Computational Neuroscience blog, June 26, 2013. http://romainbrette.fr/category/blog/what-iscomputational-neuroscience-romains-blog/. Accessed June 7, 2017.

3. Shou W, Bergstrom CT, Chakraborty AK, Skinner FK. Theory, models and biology. eLife. 2015;4:e07158.

4. The connected culture of collaboration. Digital Science Report; March 2017. https://www.digital-science.com/resources/ portfolio-reports/connected-culture-collaboration/. Accessed June 7, 2017.

5. Laurent G. What does "understanding" mean? Nat Neurosci. 2000;3:1211. 